QUARTERLY OF APPLIED MATHEMATICS

VOLUME LXVI, NUMBER 3

SEPTEMBER 2008, PAGES 437-446

S 0033-569X(08)01104-1

Article electronically published on June 4, 2008

\title{
ORTHOGONAL PROJECTIONS AND THE DYNAMICS OF CONSTRAINED MECHANICAL SYSTEMS
}

\author{
BY \\ PAULA BALSEIRO (Departmento de Matemática, Facultad de Ciencias Exactas, Universidad \\ Nacional de La Plata, Argentina) \\ AND \\ JORGE E. SOLOMIN (Departmento de Matemática, Facultad de Ciencias Exactas, Universidad \\ Nacional de La Plata and Conicet, Argentina)
}

Abstract. A coordinate-free version of the approach to mechanical systems with non-ideal restrictions developed by Udwadia (2002) and Udwadia and Kalaba (2002) in a series of articles is introduced. Some of its properties are then reinterpreted in a general geometric setting in terms of orthogonal projections. A geometric view of other aspects of constrained systems, inspired by their insight, is also presented.

1. Introduction. Some years ago, F. E. Udwadia and R. E. Kalaba 2], 4] presented a method for the study of systems with non-ideal constraints.

Their analysis of the dynamics is based on a clever decomposition of the constraining force $F^{c}$. Such a decomposition involves orthogonal projections, with respect to the inner product induced on co-vectors by the mass matrix $M$, onto subspaces associated with the virtual displacements. These orthogonal projections are implemented by means of the Moore-Penrose pseudo-inverse of a suitable matrix, a covariant procedure applicable in any coordinate system.

In this paper, we first consider Udwadia-Kalaba's method from the differential geometric point of view developed in [7] for Lagrangian mechanics. This approach will allow us to give a geometric interpretation of some of their results, in particular of the version of the Gauss principle of minimal constraint introduced in 2], and of the remarkable fact that the non-ideal component of the constraining force can be completely decoupled for writing the equations of motion [5].

Next, inspired by the orthogonal decomposition of $F^{c}$ proposed by Udwadia and Kalaba, we will consider in the last section the orthogonal projection associated with the

Received December 29, 2006.

2000 Mathematics Subject Classification. Primary 70F25, 70H45, 70G45.

Key words and phrases. Constrained mechanical systems, geometric approach.

The first author was supported in part by a fellowship of CONICET.

E-mail address: poi@mate.unlp.edu.ar

E-mail address: solo@mate.unlp.edu.ar 
inner product defined by $M$, but for tangent vectors. By doing so, we will be able to give a geometric interpretation of the classical Gauss principle of minimal constraint and of the relation between the solutions of constrained and unconstrained systems. Alternative simple proofs of that principle and of the existence and uniqueness of solutions for the dynamics of constrained systems will arise from such a geometric view. It is worth noticing that only metrics on the configuration manifold will be involved in this approach.

As in 2] or 4, we do not assume that the restrictions are linear in the velocity variables.

We believe that our approach can contribute to enlighten the deep analysis carried out by Udwadia and Kalaba and the scope of their insight.

2. The approach of Udwadia-Kalaba. Let us consider a mechanical system whose trajectories are described by

$$
q(t): R \longrightarrow R^{m}
$$

For unconstrained systems, the equations of motion are given by

$$
M \ddot{q}(t)=F(q(t), \dot{q}(t), t),
$$

where $M$ is the (symmetric positive definite) mass matrix and $F$ is the force applied on the system.

If the system is constrained by the restrictions

$$
\phi_{i}(q, \dot{q}, t)=0, \quad i=1, \ldots, k,
$$

with the $\phi_{i}$ 's smooth functions such that, at each $(q, \dot{q}, t)$, their differentials are linearly independent, Eq. (2.1) must be modified in order to take into account the force $F^{c}$ arising from the realization of the constraints:

$$
M \ddot{q}(t)=F(q(t), \dot{q}(t), t)+F^{c}(q(t), \dot{q}(t), t) .
$$

It is assumed that, at each $(q, \dot{q}, t)$ the rank of the matrix $\left(\frac{\partial \phi_{i}}{\partial \dot{q}_{j}}(q, \dot{q}, t)\right)$ is equal to $k$.

When $F^{c}$ yields a null work along every virtual displacement, the constraints are usually called ideal.

Udwadia and Kalaba [4] introduced a procedure for obtaining the equations of motion of constrained mechanical systems which encompasses the case of non-ideal constraints. Their approach is based on the decomposition of the force $F^{c}$ into two components:

$$
F^{c}=F_{i}^{c}+F_{n i}^{c} .
$$

For a brief description of this decomposition, let us consider the $k \times m$ matrix $A(q, \dot{q}, t)$ with entries

$$
A_{i j}(q, \dot{q}, t)=\frac{\partial \phi_{i}(q, \dot{q}, t)}{\partial \dot{q}_{j}} .
$$

Virtual displacements at $(q, \dot{q}, t)$ are the vectors $v$ such that

$$
A(q, \dot{q}, t) v=\mathbf{0}
$$

(see, for instance, [] $)$. 
Following Udwadia and Kalaba 4, we define the $k \times m$ matrix $B$ as

$$
B=A M^{-\frac{1}{2}}
$$

and denote by $B^{+}$its Moore-Penrose pseudo-inverse.

The forces $F_{i}^{c}$ and $F_{n i}^{c}$ in (2.4) are given by

$$
F_{i}^{c}=M^{\frac{1}{2}} B^{+} B\left(M^{-\frac{1}{2}} F^{c}\right)
$$

and

$$
F_{n i}^{c}=M^{\frac{1}{2}}\left(I-B^{+} B\right)\left(M^{-\frac{1}{2}} F^{c}\right) .
$$

REMARK 1. Any force $\tilde{F}$ yielding the same work as $F^{c}$ along virtual displacements can be taken instead of $F^{c}$ in formula (2.9). Indeed, $F_{n i}^{c}$ is defined in this way in 44. This is a very important fact: the information we need to determine $F_{n i}^{c}$ is just the work done by $F^{c}$ on virtual displacements.

Now, we recall some remarkable properties of the decomposition (2.4) 2].

Let us introduce the following notation:

$$
\begin{aligned}
& \mathcal{D}:=\operatorname{Ker} A \text { (space of virtual displacements), } \\
& \tilde{\mathcal{D}}:=\operatorname{Ker} B .
\end{aligned}
$$

It is easy to see that

$$
\tilde{\mathcal{D}}=M^{1 / 2} \mathcal{D},
$$

and that, for orthogonal complements taken with respect to the usual inner product of $R^{n}$,

$$
\tilde{\mathcal{D}}^{\perp}=M^{-1 / 2} \mathcal{D}^{\perp}
$$

Let us recall that the pseudo-inverse $B^{+}$of $B$ is determined by the following properties:

$$
\begin{aligned}
& \text { 1) } B^{+}(y)=\mathbf{0}, \forall y \in\left[B\left(R^{m}\right)\right]^{\perp}, \\
& \text { 2) } B^{+}(B x)=\Pi_{\tilde{\mathcal{D}}^{\perp}} x,
\end{aligned}
$$

with $\Pi_{\tilde{\mathcal{D}}^{\perp}}$ the orthogonal projection onto $\tilde{\mathcal{D}}^{\perp}$.

Since we are assuming $\operatorname{rank}(A)=k$, we have $B\left(R^{m}\right)=R^{k}$. Then, (2.14) is meaningless for the matrix $B$. Consequently, $B^{+}$is characterized just by (2.15).

Thus, $B^{+} B\left(M^{-1 / 2} F^{c}\right)$ and $\left(I-B^{+} B\right)\left(M^{-1 / 2} F^{c}\right)$ yield the orthogonal decomposition of $M^{-1 / 2} F^{c}$ into its components in $\tilde{\mathcal{D}}^{\perp}$ and $\tilde{\mathcal{D}}$.

So, taking into account (2.8) and (2.9), we have

$$
\begin{gathered}
F_{i}^{c} \in M^{1 / 2} \tilde{\mathcal{D}}^{\perp}, \\
F_{n i}^{c} \in M^{1 / 2} \tilde{\mathcal{D}} .
\end{gathered}
$$

Because of (2.12) and (2.13), we have that (2.16) and (2.17) are equivalent to P1: For every $F^{c}$, the work of $F_{i}^{c}$ along any virtual displacement vanishes, whereas the acceleration produced by $F_{n i}^{c}$ belongs to $\mathcal{D}[2]$.

We shall consider, besides the usual norm of $R^{m}$, the inner product defined by the matrix $M=\left(m_{i j}\right)$ on vectors: for $u$ and $v$ with components $\left(u^{i}\right)$ and $\left(v^{j}\right)$,

$$
\langle u, v\rangle_{M}:=\sum m_{i j} u^{i} v^{j} .
$$


The metric induced by $\langle,\rangle_{M}$ on covectors (forces) is the following: for $F$ and $G$ with components $F_{i}$ and $G_{j}$, if $M^{-1}=\left(m^{i j}\right)$,

$$
\begin{aligned}
\langle F, G\rangle_{\text {induced by }\langle,\rangle_{M}} & =\langle\beta(F), \beta(G)\rangle_{M} \\
& =\sum m_{i j} \beta(F)^{i} \beta(G)^{j},
\end{aligned}
$$

where $\beta(F)=\sum \beta(F)^{i} \frac{\partial}{\partial x^{i}}$, with $\beta(F)^{i}=\sum m^{i j} F_{j}, m^{i j}$ being the entries of $M^{-1}$, and analogously for $\beta(G)$.

The components of the vector $\beta(F)$ are obtained from those of $F$ by raising its indexes by means of the matrix $M^{-1}$. So, it is clear that it represents the acceleration produced by $F$. It is also clear that the work of a force $F$ along a vector $v$ turns out to be $\beta(F)(v)=\langle\beta(F), v\rangle_{M}$.

In particular, the norm of $F$ for this metric is given by

$$
\begin{aligned}
\|F\|_{M^{-1}} & =\left(\langle F, F\rangle_{M^{-1}}\right)^{1 / 2} \\
& =\left(\sum \frac{F_{i}^{2}}{m_{i}}\right)^{1 / 2} \\
& =\|\beta(F)\|_{M} .
\end{aligned}
$$

In this context, $\mathbf{P} \mathbf{1}$ yields

$$
\left\langle F_{i}^{c}, F_{n i}^{c}\right\rangle_{M^{-1}}=\left\langle F_{i}^{c}, M^{-1} F_{n i}^{c}\right\rangle=0,
$$

with $\langle$,$\rangle the usual inner product of R^{m}$.

That is,

P2: The forces $F_{i}^{c}$ and $F_{n i}^{c}$ are orthogonal for $\langle., .\rangle_{M^{-1}}$. Equivalently, the accelerations $\beta\left(F_{i}^{c}\right)$ and $\beta\left(F_{n i}^{c}\right)$ produced by $F_{i}^{c}$ and $F_{n i}^{c}$ respectively, are orthogonal for $\langle., .\rangle_{M}[5]$.

REMARK 2. It is worth noticing that (2.8) and (2.9) represent a clever way of expressing $M^{-1}$-orthogonal projections in terms of the Moore-Penrose pseudo-inverses associated with the usual norm of $R^{n}$.

In terms of $A^{+_{M}}$, the pseudo-inverse of the matrix $A$ associated with the inner product $\langle,\rangle_{M}$ in $R^{m}, F_{i}^{c}$ and $F_{n i}^{c}$ can be written as

$$
\begin{gathered}
F_{i}^{c}=M A^{+_{M}} A M^{-1}\left(F^{c}\right), \\
F_{n i}^{c}=M\left(I-A^{+_{M}} A M^{-1}\right)\left(F^{c}\right) .
\end{gathered}
$$

(As before, since we are assuming $\operatorname{rank}(A)=k$, the $m \times k$ matrix $A^{+_{M}}$ is characterized by the property

$$
A^{+M}(A x)=\Pi_{\mathcal{D}^{\perp}}^{M} x,
$$

with $\Pi_{\mathcal{D}^{\perp}}^{M}$ the orthogonal projection onto $\mathcal{D}^{\perp}$ with respect to $\langle,\rangle_{M}$.) See [2], 4].

Another important property of Udwadia-Kalaba's approach, proved in [2], is the $E x$ tended Gauss Principle of minimal constraint. For ideal restrictions, the Gauss Principle of minimal constraint asserts that the norm $\left\|F^{c}\right\|_{M^{-1}}$ of the constraining force $F^{c}$ is the minimum of the set

$$
\left\{\|G\|_{M^{-1}} \text { for } G \in C\right\},
$$


where

$$
C=\left\{G \text { s.t. the system with } \ddot{q}(t)=M^{-1}(F+G) \text { satisfies the constraints }\right\} .
$$

P3: The norm $\left\|F_{i}^{c}\right\|_{M^{-1}}$ is the minimum of the set

$$
\left\{\|G\|_{M^{-1}} \text { s.t. } G \in \tilde{C}\right\}
$$

with

$$
\tilde{C}=\left\{G \text { s.t. the system with } \ddot{q}(t)=M^{-1}\left(F+F_{n i}^{c}+G\right) \text { satisfies the constraints }\right\} .
$$

3. Lagrangian mechanics in an invariant form. In this section, we recall the geometric framework introduced in [7] for the Lagrangian mechanics and analyze the relation between admitted generators of the dynamics and virtual displacements for constrained systems.

Let us consider a mechanical system having the $n$-dimensional manifold $Q$ as its configuration space and

$$
L(q, v): T Q \rightarrow R
$$

as a Lagrangian.

It will be assumed that the symmetric matrix

$$
M=\left(M_{i j}\right)_{i, j=1, \ldots, n},
$$

with

$$
M_{i j}:=\frac{\partial^{2} L}{\partial v^{i} \partial v^{j}},
$$

is positive definite at every $(q, v) \in T Q$.

Thus, the matrix $M$ defines an interior product $\langle,\rangle_{M}$ on $T_{q} Q$ at each $q \in Q$.

The application $\beta$ from covectors to vectors defined in section 2 generalizes to this framework: $\beta$ is now the isomorphism from $T_{q}^{*} Q$ onto $T_{q} Q$ defined through the equality

$$
\langle u, \beta(\alpha)\rangle_{M}=\alpha(u) \quad \forall u \in T_{q} Q .
$$

Notice that $\forall \alpha_{1}, \alpha_{2} \in T_{q}^{*} Q$,

$$
\left\langle\beta\left(\alpha_{1}\right), \beta\left(\alpha_{2}\right)\right\rangle_{M}=\left\langle\alpha_{1}, \alpha_{2}\right\rangle_{M^{-1}} .
$$

The Lagrangian form associated with $L$ is the symplectic form $\omega_{L}$ on $T Q$ defined by

$$
\omega_{L}:=-\left(\frac{\partial^{2} L}{\partial v^{i} \partial v^{j}}\right) d q^{i} \wedge d v^{j}+\left(\frac{\partial^{2} L}{\partial v^{i} \partial q^{j}}\right) d q^{i} \wedge d q^{j} .
$$

Let us denote

$$
\mathcal{X}(T Q):=\{\text { vector fields on } T Q\} .
$$

In this context, forces are represented by the horizontal 1-forms on $T Q$, i.e. 1-forms vanishing on vectors which are tangent to the fibers. The horizontal 1-forms on $T Q$ are canonically identified with the 1-forms on $Q$ in an obvious way. We will sometimes look at 1-forms on $Q$ as horizontal 1-forms on $T Q$ and vice versa. By using this identification, we see that the work a force $F$ does on a vector $v$ tangent to $Q$ is just $F(v)$. 
The dynamics of the (unconstrained) system, when an external force $F^{e}$ is acting, is generated by the unique $X_{U} \in \mathcal{X}(T Q)$ satisfying

$$
\omega_{L}(q, v)\left(X_{U}(q, v),(u, w)\right)+d E_{L}(u, w)=F^{e}(u, w), \quad \forall(u, w) \in T_{(q, v)}(T Q),
$$

where $E_{L}(q, v)=\frac{\partial L}{\partial v} \cdot v-L(q, v)$ is the energy function associated with $L$ [7].

The existence and uniqueness of $X_{U}$ follows from the fact that $\omega_{L}$ is symplectic.

A direct computation shows that $X_{U}$ is a vector field defining a second-order equation. That is, if $\pi: T Q \rightarrow Q$ is the canonical projection $\pi(q, v)=q$, then $\pi_{*}(X(q, v))=v$. As in [7, we will call special vector fields the elements of $\mathcal{X}(T Q)$ having this property.

Let us denote

$$
\mathcal{S}(T Q):=\{X \in \mathcal{X}(T Q) \text { s.t. } X \text { is special }\} .
$$

Notice that only special vector fields on $T Q$ have a physical meaning. In fact, the possible trajectories $q(t)$ are lifted to $T Q$ as $(q(t), \dot{q}(t))$ and so, only special vectors can be tangent to them.

We recall that a vector $(u, w) \in T_{(q, v)} T Q$ is called vertical if $\pi_{*}(u, w)=0$; i.e, $u=\mathbf{0}$. In local coordinates $\left(q^{i}, v^{i}\right)$, a vertical tangent vector has the form $u^{i} \frac{\partial}{\partial v^{i}}$.

We will denote by $\mathcal{V}(T Q)$ the space of vertical vector fields on $T Q$ and by $\tau$ the canonical isomorphism from $\mathcal{X}(Q)$ onto $\mathcal{V}(T Q)$. That is, for $Y \in \mathcal{X}(Q)$,

$$
\tau(Y)=(\mathbf{0}, Y) \in \mathcal{V}(T Q) .
$$

Now, let us come back to the restrictions

$$
(q(t), \dot{q}(t)) \in \mathcal{C},
$$

with $\mathcal{C}$ a submanifold of $T Q$ locally defined as the zeros of $k$ smooth functions

$$
\phi^{i}(q, v)=0, \quad i=1, \ldots, k .
$$

As in [7, we will assume that the constraint is admissible; i.e., at each $(q, v) \in \mathcal{C}$,

$$
\operatorname{dim}\left(\operatorname{span}\left\{\frac{\partial \phi^{i}}{\partial v^{j}} d v^{j}(q, v)\right\}\right)=\operatorname{dim}\left(\operatorname{span}\left\{d \phi^{i}(q, v)\right\}\right)=k .
$$

REMARK 3. We consider Lagrangians and constraints independent of time just for simplicity: the analysis for time-dependent ones is completely analogous.

The set of generators of dynamics which are compatible with the restrictions is

$$
\mathcal{S}(\mathcal{C}):=\left\{X \in \mathcal{S}(T Q) \text { s.t. } d \phi^{i}(X)=0, i=1, \ldots, k\right\},
$$

the set of special vector fields belonging to $\mathcal{X}(\mathcal{C})$. On the other hand, the space of virtual displacements is given, at each $(q, v) \in \mathcal{C}$, by (see for instance [1] or [6])

$$
\mathcal{D}_{(q, v)}:=\left\{u \in T_{q} Q \text { s.t. } d \phi^{i}(q, v) . \tau(u)=0, i=1, \ldots, k\right\} .
$$

If, as in the previous sections, we denote by $A$ the matrix $\left(\frac{\partial \phi^{i}}{\partial v^{j}}\right)$, the space of virtual displacements at $(q, v)$ can be expressed as

$$
\mathcal{D}_{(q, v)}:=\left\{u \in T_{q} Q \text { s.t. } A(q, v) u=0\right\} .
$$

We will write $\mathcal{D}_{q}$ instead of $\mathcal{D}_{(q, v)}$ when it only depends on $q \in Q$, and analogously for other distributions. 
Remark 4. At each $(q, v) \in \mathcal{C}$, if $(v, u) \in \mathcal{S}(\mathcal{C})_{(q, v)}$, then

$$
(v, w) \in \mathcal{S}(\mathcal{C})_{(q, v)} \Leftrightarrow \tau^{-1}((v, u)-(v, w)) \in \mathcal{D}_{(q, v)} .
$$

In fact, since $(v, u),(v, w) \in T_{(q, v)}(T Q)$ are special vectors and $d \phi^{i}(v, u)=0$ for $i=1, \ldots, k$, then

$$
\begin{aligned}
(v, w) \in \mathcal{S}(\mathcal{C})_{(q, v)} & \Leftrightarrow d \phi^{i}(v, w)=0, \quad i=1, \ldots, k \\
& \Leftrightarrow d \phi^{i}[(v, u)-(v, w)]=0, \quad i=1, \ldots, k \\
& \Leftrightarrow A(q, v)(u-w)=0 .
\end{aligned}
$$

Given that $\tau^{-1}((v, u)-(v, w))=u-w$, we obtain equation (3.13).

Thus, $\mathcal{S}(\mathcal{C})_{(q, v)}$ is an affine subspace of $T_{(q, v)}(T Q)$ and $\mathcal{D}_{(q, v)}$ is the vector subspace associated with it.

It is shown in [7] that, under our assumptions, $\mathcal{S}(\mathcal{C})_{(q, v)}$ is not empty.

The dynamics of the restricted system is generated by the unique vector field $X_{R} \in$ $\mathcal{S}(\mathcal{C})$ satisfying, at each $(q, v) \in \mathcal{C}$,

$$
\omega_{L}\left(X_{R}(q, v),(u, w)\right)+d E_{L}(u, w)=\left(F^{e}+F^{c}\right)(u, w), \quad \forall(u, w) \in T_{(q, v)}(T Q),
$$

where $F^{c}$ is the force exerted by the constraints in order for the restrictions to be satisfied and, as above, $F^{e}$ is the external force.

Taking into account that $F^{e}$ and $F^{c}$ are horizontal 1-forms on $T Q$, by identifying them with 1- forms on $Q$ we can rewrite (3.14) as

$$
\omega_{L}\left(X_{R}(q, v),(u, w)\right)+d E_{L}(u, w)=\left(F^{e}+F^{c}\right)(u), \quad \forall(u, w) \in T_{(q, v)}(T Q) .
$$

In any coordinate patch, (3.15) is equivalent to the Euler-Lagrange equations

$$
\left(M_{i j} \ddot{q}^{j}+\frac{\partial^{2} L}{\partial \dot{q}^{i} \partial q^{j}} \dot{q}^{j}-\frac{\partial L}{\partial q^{i}}\right)\left(u^{i}\right)=\left(F_{i}^{e}+F_{i}^{c}\right)\left(u^{i}\right) \quad \forall u \in T_{q} Q .
$$

In section 5 , we will give a very simple geometric proof of the existence and uniqueness of the vector field $X_{R}$ satisfying (3.14) and a geometric interpretation of the Gauss principle of minimal constraint.

Notice that, with our notation, the classical Gauss principle of minimal constraint can be written as:

$X_{R}$ is the vector field in $\mathcal{S}(\mathcal{C})$ satisfying

$$
\left\|\beta^{-1}\left(\tau^{-1}\left(X_{R}-X_{U}\right)\right)\right\|_{M^{-1}}=\min _{X \in \mathcal{S}(\mathcal{C})}\left\|\beta^{-1}\left(\tau^{-1}\left(X-X_{U}\right)\right)\right\|_{M^{-1}},
$$

or, equivalently,

$$
\left\|\tau^{-1}\left(X_{R}-X_{U}\right)\right\|_{M}=\min _{X \in \mathcal{S}(\mathcal{C})}\left\|\tau^{-1}\left(X-X_{U}\right)\right\|_{M}
$$

4. Udwadia-Kalaba's method revisited. For each $(q, v) \in \mathcal{D}$, we denote by

$$
P_{\beta^{-1} \mathcal{D}}: T_{q}^{*} Q \rightarrow \beta^{-1}\left(\mathcal{D}_{(q, v)}\right)
$$

the $M^{-1}$-orthogonal projection.

It follows from section 2 that in the Udwadia-Kalaba decomposition

$$
F^{c}=F_{i}^{c}+F_{n i}^{c},
$$


$F_{n i}^{c}$ turns out to be equal to $P_{\beta^{-1} \mathcal{D}}\left(F^{c}\right)$.

As mentioned in Remark 1, this implies

$$
\beta\left(F_{n i}^{c}\right) \in \mathcal{D} .
$$

So, from Remark 4, we have

$$
X_{U}+\tau\left(\beta\left(F_{i}^{c}\right)\right) \in \mathcal{S}(\mathcal{C}) .
$$

Thus, we have the following remarkable property of decomposition (4.1), which is rather implicit in 4] or [2]:

Property 1. The dynamics of the restricted system is generated by the vector field

$$
X_{R}=X_{R}^{i}+\tau\left(\beta\left(F_{n i}^{c}\right)\right),
$$

where $X_{R}^{i}=X_{U}+\tau\left(\beta\left(F_{i}^{c}\right)\right)$ would generate the dynamics of the system if the restriction were ideal.

Since $F_{n i}^{c}=P_{\beta^{-1}} \mathcal{D}(\tilde{F})$ for any force $\tilde{F}$ making the same work as $F^{c}$ on the virtual displacement, from Property 1 we have

Property 2. The dynamics of the restricted system is generated by the vector field

$$
X_{R}=X_{R}^{i}+\tau\left(\beta\left(P_{\beta^{-1} \mathcal{D}}\right)(\tilde{F})\right),
$$

for any force $\tilde{F}$ such that $\tilde{F}(u)=F^{c}(u) \forall u \in \mathcal{D}$.

Now, taking into account that, since $X_{R}^{i}-X_{U}$ is vertical, we can give the following alternative version of the Gauss principle of minimal action presented in 2]:

Property 3. For any force $\tilde{F}$ such that $\tilde{F}(u)=F^{c}(u) \forall u \in \mathcal{D}$, the vector field $X_{R}$ can be written as

$$
X_{R}=X_{R}^{i}+\tau\left(\beta\left(P_{\beta^{-1} \mathcal{D}}\right)(\tilde{F})\right),
$$

with $X_{R}^{i} \in \mathcal{S}(\mathcal{C})$ satisfying

$$
\left\|\tau^{-1}\left(X_{R}^{i}-X_{U}\right)\right\|_{M}=\min _{X \in \mathcal{S}(\mathcal{C})}\left\|\tau^{-1}\left(X-X_{U}\right)\right\|_{M} .
$$

In fact, according to the classical Gauss principle, this equation characterizes the generator of the dynamics for the ideal case, and then Property 3 follows from Property 2 .

(As mentioned before, a simple proof of the classical Gauss principle will be included in the next section.)

5. Restricted systems and $M$-orthogonal projections. In this section, we will consider the $M$-orthogonal projection of tangent vectors onto $\mathcal{D}$. It will be used for obtaining a geometric interpretation and simple proofs of the existence and uniqueness of $X_{R}$ and the Gauss principle of minimal constraint.

For each $(q, v) \in \mathcal{C}$, we will denote by $\Pi_{\mathcal{D}}$ the $M$-orthogonal projection

$$
\Pi_{\mathcal{D}}: T_{q} Q \rightarrow \mathcal{D}_{(q, v)} .
$$

REMARK 5. The projection $\Pi_{\mathcal{D}}$ is related to $P_{\beta^{-1} \mathcal{D}}$ through the identity

$$
\Pi_{\mathcal{D}}(\beta(\alpha))=\beta\left(P_{\beta^{-1} \mathcal{D}}(\alpha)\right), \quad \forall \alpha \in T_{q}^{*} Q .
$$


We will consider the ideal case. The results can be easily extended to the non-ideal one by applying Property 1 of the previous section.

Let us take a fixed $X_{0} \in \mathcal{S}(\mathcal{C})$.

Notice that, for $X_{U} \in \mathcal{S}(T Q)$ and $X_{R} \in \mathcal{S}(\mathcal{C}), X_{U}-X_{0}$ and $X_{R}-X_{0}$ are vertical vector fields. So, we can write

$$
X_{U}=X_{0}+\tau\left(Y_{U}\right)
$$

and

$$
X_{R}=X_{0}+\tau\left(Y_{R}\right)
$$

where, according to Remark $4, Y_{R} \in \mathcal{D}$.

A simple characterization of $X_{R}$ in terms of $X_{U}$ is given in the following

Proposition 1. In the case of ideal restrictions, for $Y_{U}$ and $Y_{R}$ as in (5.1) and (5.2), it follows that

$$
Y_{R}=\Pi_{\mathcal{D}}\left(Y_{U}\right) .
$$

Proof. From (3.7) and (3.14), we see that $X_{R}$ is characterized by two properties:

a) $X_{R} \in \mathcal{S}(\mathcal{C})$;

b) at each $(q, v) \in \mathcal{C}$,

$$
\omega_{L}\left(X_{U}-X_{R},(u, w)\right)=0, \quad \forall(u, w) \in T_{(q, v)} \mathcal{C} \text { s.t. } u \in \mathcal{D}_{(q, v)} .
$$

But, from the definition (3.5) of $\omega_{L}$, since

$$
\left(\frac{\partial^{2} L}{\partial v^{i} \partial q^{j}}\right) d q^{i} \wedge d q^{j}\left(\left(\mathbf{0}, Y_{U}-Y_{R}\right),(u, w)\right)=0,
$$

we have

$$
\begin{aligned}
\omega_{L}\left(X_{U}-X_{R},(u, w)\right) & =-\left(\frac{\partial^{2} L}{\partial v^{i} \partial v^{j}}\right) d q^{i} \wedge d v^{j}\left(\left(\mathbf{0}, Y_{U}-Y_{R}\right),(u, w)\right) \\
& =\left(\frac{\partial^{2} L}{\partial v^{i} \partial v^{j}}\right)\left(Y_{U}-Y_{R}\right)^{i} u^{j}
\end{aligned}
$$

Thus,

$$
\omega_{L}\left(X_{U}-X_{R},(u, w)\right)=\left\langle Y_{R}(q, v)-Y_{U}(q, v), u\right\rangle_{M} .
$$

Then, taking into account Remark 4 a) and b) hold if and only if $Y_{R}=\Pi_{\mathcal{D}}\left(Y_{U}\right)$.

Notice that equation (5.5), and consequently the previous proposition, are independent of the particular $X_{0} \in \mathcal{S}(\mathcal{C})$ chosen.

This proposition can also be seen as an alternative geometric proof of the existence and uniqueness of $X_{R}$.

Moreover, it also gives rise to a simple geometric proof of the

Gauss principle of minimal constraint. For ideal restrictions, the vector field $X_{R} \in$ $\mathcal{S}(\mathcal{C})$ generating the dynamics of the constrained system is characterized by

$$
\left\|\tau^{-1}\left(X_{R}-X_{U}\right)\right\|_{M}=\min _{X \in \mathcal{S}(\mathcal{C})}\left\|\tau^{-1}\left(X-X_{U}\right)\right\|_{M} .
$$

Proof. Since $X \in \mathcal{S}(\mathcal{C})$ if and only if

$$
X=X_{0}+\tau(Y),
$$


with $Y \in \mathcal{D}$, then, $\forall X \in \mathcal{S}(\mathcal{C})$,

$$
\begin{aligned}
\left\|\tau^{-1}\left(X-X_{U}\right)\right\|_{M} & =\left\|Y-Y_{U}\right\|_{M} \\
& \geq\left\|Y_{U}-\Pi_{\mathcal{D}}\left(Y_{U}\right)\right\|_{M} \\
& =\left\|\tau^{-1}\left(X_{R}-X_{U}\right)\right\|_{M} .
\end{aligned}
$$

It is clear that the equality only holds for $X=X_{R}$.

REMARK 6. In the framework of [4] or [2] recalled in section 2, the projection $\Pi_{\mathcal{D}}$ can be represented by means of Moore-Penrose pseudo-inverses with respect to the usual norm on $R^{n}$ as

$$
\Pi_{\mathcal{D}}(Y)=M^{-\frac{1}{2}}\left(I-B^{+} B\right) M^{\frac{1}{2}}(Y),
$$

with, as above, $B=A M^{-\frac{1}{2}}$.

As was done in section 2, the projection $\Pi_{\mathcal{D}}$ can also be written in terms of $A^{+_{M}}$, the pseudo-inverse of the matrix $A$ associated with $\langle,\rangle_{M}$ :

$$
\Pi_{\mathcal{D}}(Y)=\left(I-A^{+M} A\right)(Y) .
$$

\section{REFERENCES}

[1] J.E. Solomin and M. Zuccalli, A geometric approach to the extended D'Alembert principle of Udwadia-Kalaba-Hee-Chang. Quart. Appl. Math. 63, 269-275 (2005). MR2150773 (2006a:70043)

[2] F.E. Udwadia, Fundamental principles and Lagrangian dynamics: Mechanical systems with nonideal, holonomic, and nonholonomic constraints. J. Math. Anal. Appl. 251, 341 (2002). MR.1790412 (2001j:70014)

[3] F.E. Udwadia, On Constrained Motion. Applied Mathematics and Computation. 164, 313-320 (2005). MR2131158 (2005m:70071)

[4] F.E. Udwadia and R.E. Kalaba, On the foundations of analytical dynamics. Internat. J. Non-linear Mech. 37, 1079-1090 (2002), and references therein. MR.1897289 (2003f:70023)

[5] F.E. Udwadia and R.E. Kalaba, What is the General Form of the Explicit Equations of Motion for Constrained Mechanical Systems? Journ. Applied Mechanics, 69, 335-339 (2002). MR2000941 (2004g:70038)

[6] F.E. Udwadia, R.E. Kalaba, E. Hee-Chang, Equations of motion for constrained mechanical systems and the extended D'Alembert principle. Quart. Appl. Math. (LV) 2, 321-331 (1997). MR1447580 (98f:70016)

[7] A.M. Vershik, Classical and non-classical dynamics with constraints. Lecture Notes in Mathematics 1108, 278-301, Springer-Verlag 1984. 